\title{
Roughness Effects on Wind-Turbine Wake Dynamics in a Boundary-Layer Wind Tunnel
}

\author{
Barlas, Emre; Buckingham, Sophia ; van Beeck, Jeroen
}

\section{Published in:}

Boundary-Layer Meteorology

Link to article, DOI:

$10.1007 / \mathrm{s} 10546-015-0083-z$

Publication date:

2016

Document Version

Peer reviewed version

Link back to DTU Orbit

Citation (APA):

Barlas, E., Buckingham, S., \& van Beeck, J. (2016). Roughness Effects on Wind-Turbine Wake Dynamics in a Boundary-Layer Wind Tunnel. Boundary-Layer Meteorology, 158(1), 27-42. https://doi.org/10.1007/s10546-015$0083-z$

\section{General rights}

Copyright and moral rights for the publications made accessible in the public portal are retained by the authors and/or other copyright owners and it is a condition of accessing publications that users recognise and abide by the legal requirements associated with these rights.

- Users may download and print one copy of any publication from the public portal for the purpose of private study or research.

- You may not further distribute the material or use it for any profit-making activity or commercial gain

- You may freely distribute the URL identifying the publication in the public portal 


\title{
Roughness Effects on Wind-Turbine Wake Dynamics in a Boundary-Layer Wind Tunnel
}

\author{
E. Barlas · S. Buckingham • J. van Beeck
}

Received: date / Accepted: date

\begin{abstract}
Increasing demand in wind energy has resulted in increasingly clustered wind farms, and raised the interest in wake research dramatically in the last couple of years. To this end, the present work employs an experimental approach with scaled three-bladed wind-turbine models in a large boundary-layer wind-tunnel. Time-resolved measurements are carried out with threecomponent hot-wire anemometer in the mid-vertical plane of the wake up to a downstream distance of eleven turbine diameters. The major issue addressed is the wake dynamics i.e. the flow and turbulence characteristics as well as spectral content under two different neutral boundary-layer inflow conditions. The wind tunnel is arranged with and without roughened surfaces in order to mimic moderately rough and smooth conditions. The inflow characterization is carried out by using all three velocity components, while the rest of the study is focused on the streamwise component's evolution. The results show an earlier wake recovery, i.e. the velocity deficit due to the turbine is less persistent for the rough case due to higher incoming turbulence levels. This paves the way for enhanced mixing from higher momentum regions of the boundary layer towards the centre of the wake. The investigation on the turbulent shear stresses is in line with this observation as well. Moreover, common as well as distinguishing features of the turbulent-scales evolution are detected for rough and smooth inflow boundary-layer conditions. Wake meandering disappears for rough inflow conditions but persists for smooth case with a Strouhal number similar to that of a solid disk wake.
\end{abstract}

Keywords Roughness effects · Three-component hot-wire anemometer · Wind-tunnel experiment · Wind-turbine wakes

\section{Introduction}

The number of wind farms worldwide is increasing resulting in turbines that are situated in an increasingly clustered manner. This grouping gives rise to two main disadvantages in terms of

Emre Barlas $(\varangle)$

DTU Wind Energy, Technical University of Denmark, 2800 Kongens Lyngby, Denmark

Tel.: +45-45254340

E-mail: ebarlas@dtu.dk

Sophia Buckingham · Jeroen van Beeck

Environmental and Applied Fluid Dynamics Department, von Karman Institute for Fluid Dynamics, Chaussée de Waterloo 72, B-1640, Rhode-St-Genèse, Belgium

E-mail: sophia.buckingham@vki.ac.be

E-mail: vanbeeck@vki.ac.be 
cost of energy. First, a wind turbine operating in the wake has a reduced power production due to lower incident wind speed. Secondly, the large-scale and small-scale structures in the wake results in added turbulence that decreases the lifetime of downstream turbines due to increased fatigue. These are two well-known issues for wind-farm wake aerodynamics. Nevertheless a deep enough understanding has not been fully reached, as illustrated by relatively poorly performing computational or analytical models (Crespo et al. 1999; Sanderse et al. 2011; Gaumond et al. 2012).

The shortcomings of the models are mostly due to lack of understanding of the complex interaction between the atmospheric boundary layer and wind turbine, as pointed out by Rados et al. (2009). Additionally, the unsteady nature of the wake gives rise to other problems such as the meandering phenomenon (Bingol et al., 2010). In simplest terms, it is the movement of the wake as a whole in both the horizontal and vertical directions as the wake is convected downstream. This transport process is modelled by Larsen et al. (2008) assuming the wakes to act as passive tracers driven by the large-scale turbulence structures. This does not necessarily yield a periodic behaviour. However, there are studies in the literature that relate the meandering behaviour to the intrinsic instabilities as in bluff-body vortex shedding (Medici and Alfredsson, 2006), even though this relation has been contradicted by Devinant et al. (2011) and Larsen et al. (2008).

The aim of this work is to provide additional data to the existing experimental wake database with tailored-designed rotating turbine models and state-of-the-art experimental techniques in order to extract a physical understanding of how boundary-layer turbulence affects the spatial wake development. In addition to this, the meandering phenomenon is investigated with respect to the evolution of turbulent length scales through spectral analysis.

\section{Experimental Set-up}

2.1 Wind-Turbine Model

The three-bladed wind-turbine model has a 150-mm diameter and a 130-mm hub height and is representative of a scaled $2 \mathrm{MW}$ offshore wind turbine (see Fig. 1).

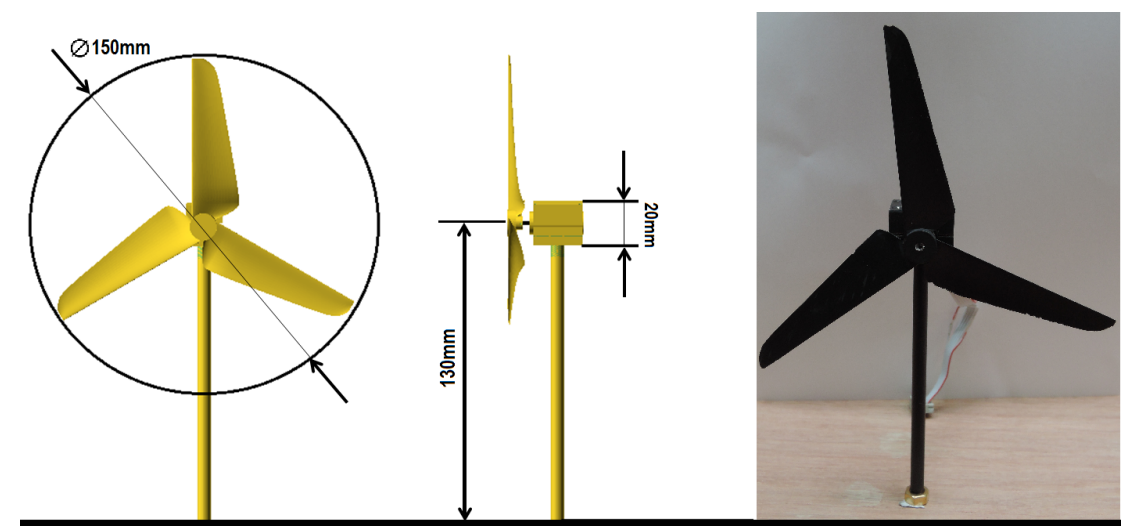

Fig. 1 Wind-turbine model dimensions and front view.

The working principle of the model is the following: a small direct current (DC) motor inside the motor housing (nacelle) is used as a generator. It is connected to an electrical circuit with which it can be counter-loaded in order to extract power from the airflow. This process does not only extract power, but also decreases the turbine rotational speed, which is tracked via the encoder 
mounted to the motor. Thereby the tip-speed ratio, $\lambda,\left(\lambda=\omega \cdot r / U_{h}\right.$ where $\omega$ is the angular velocity, $r$ is the rotor radius and $U_{h}$ is the hub-height speed) can be observed and controlled with the variable resistance in the circuit.

The tip-speed ratio is an important parameter, since the thrust of the turbine is directly related to it. The characterization of the model, namely the relationship of the electrical resistance versus tip-speed ratio, was carried out before the experiments. Since there were no force measurements, comparative studies with two different boundary-layer conditions were carried out under same tipspeed ratio $(\lambda=5)$. Thereby, the aim has been to concentrate solely on the boundary-layer effects on the wind-turbine wake, while the thrust remained constant.

Moreover, the blades were designed based on blade element momentum theory considering the low Reynolds numbers $\left(\approx 10^{5}\right)$ that is reached and for a design tip-speed ratio of 5 . During the design process a thin airfoil section GM15 was used and manufactured via three-dimensional printing. A detailed analysis of the blade design can be found in Bossuyt (2013). It is worthwhile mentioning that the wind-turbine wake studies that are carried out in wind tunnels with miniature wind turbines can result in misleading conclusions because of the large scaling ratios $(\approx 1: 500)$. In other words, the experiments that are conducted at lower Reynolds numbers, to full scale conditions may affect the flow statistics. However, according to Chamorro et al. (2012) the Reynolds number independence (on the basis of the rotor diameter and hub-height speed) for the higher order statistics (i.e. turbulence intensity and kinematic shear stress) was reached for values over $9.3 \times$ $10^{4}$, while this value was even lower for the mean speed statistics $\left(\approx 4.8 \times 10^{4}\right)$. The present set of experiments were carried out at a hub-height speed of $8 \mathrm{~m} \mathrm{~s}^{-1}$. Based on the turbine diameter of $0.15 \mathrm{~m}$, the reached Reynolds number was $8.5 \times 10^{4}$.

\subsection{Experimental Facility and Measurement Technique}

The experiments were carried out at the VKI-L1B wind tunnel. The closed return circuit tunnel has a contraction ratio of 4.7 and is powered by a variable-speed DC motor of $580 \mathrm{~kW}$ driving two contra rotation fans of $4.2 \mathrm{~m}$ diameter. The L1B configuration is of interest for wind-engineering applications among other tunnel settings. In this configuration the rectangular test section is $2 \mathrm{~m}$ high, $3 \mathrm{~m}$ wide and $20 \mathrm{~m}$ long with the possibility of a roughened floor to allow for the growth of a scaled down turbulent boundary layer similar in nature to the lower part of the neutral atmospheric boundary layer. The end of the test section is equipped with a $2.6 \mathrm{~m}$ diameter turn table on which the model to be tested is placed.

The measurement probe used during the campaigns was a three-component Dantec 55P91 hotwire anemometer (3C-HWA). In addition to the standard velocity calibration the 3C-HWA requires also a one-time directional calibration. This is important in order to decompose the velocity into its components correctly. The directional sensitivity of tri-axial probes is characterized by both a yaw and a pitch coefficient. In this study, the coefficients that were calculated via the calibration performed by Fruytier (1993) were used. For a more detailed description of the calibration and decomposition of the velocity components procedure, refer to Annex A of Conan (2012). The 3CHWA is indeed an important tool to acquire all three components in a time-resolved manner. On the other hand, it might be disadvantageous, since the spatial resolution is lower in comparison to a single component anemometer and the measurements are only reliable for flow within \pm 35 degree of the cone. This is an important factor that one should keep in mind during the data acquisition and post processing periods.

The measurements were taken at the mid vertical plane of the wake $(x / D=-1,1,2,3,4,5,7$, 9,11 ; where $x$ and $D$ is the streamwise coordinate and the turbine diameter, respectively). At each downstream location 28 measurement points were covered vertically. Starting from 30-mm above ground $\left(z / z_{\text {tip }} \approx 0.15\right.$ where $z$, the vertical coordinate is normalized with $z_{\text {tip }}$, the height at the top tip of the turbine model) to $260-\mathrm{mm}\left(z / z_{\text {tip }} \approx 1.3\right)$ the points are separated with increments 
a)
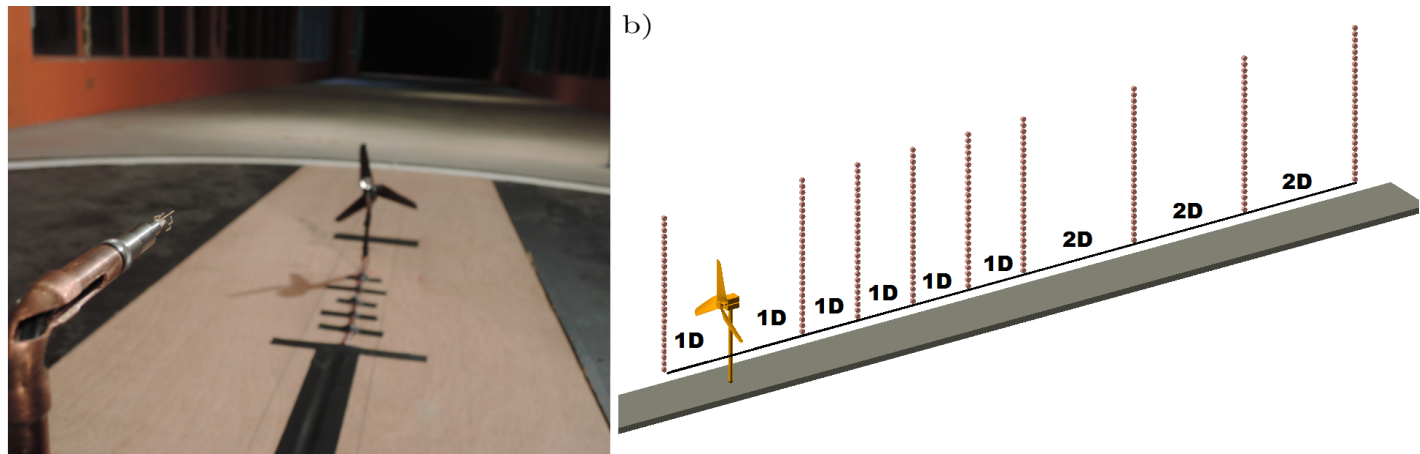

Fig. 2 a) Three-component HWA set-up installed in VKI L1-B wind tunnel test section. b) Dots representing measurement points at various downstream locations expressed in turbine diameter (D).

of $10-\mathrm{mm}$ and above that until $340-\mathrm{mm}\left(z / z_{\text {tip }} \approx 1.7\right)$ the distances were $20-\mathrm{mm}$. At each point the measurements were made at $3 \mathrm{kHz}$, for at least 120 seconds and filtered at $1 \mathrm{kHz}$. In Fig. 2b, each point represents the measurement positions for the 3C-HWA instrument.

During the uncertainty analysis, bias and random errors were taken into account. However, considering the long enough observation time the random error is expected to be low. The bias error was calculated first considering a single component hot wire and then this was assumed to be valid for all three components. Subsequently, the errors that are caused by the rotation and Jørgensen matrices, are added together (see Fig. 3). The propagation was done by estimating a $2^{\circ}$ variation on the angles used in the rotational matrix and $5 \%$ error on pitch and yaw coefficients. Overall, this leads to an error of $7 \%$ for the velocity range of interest; 7 to $9 \mathrm{~m} \mathrm{~s}^{-1}$.

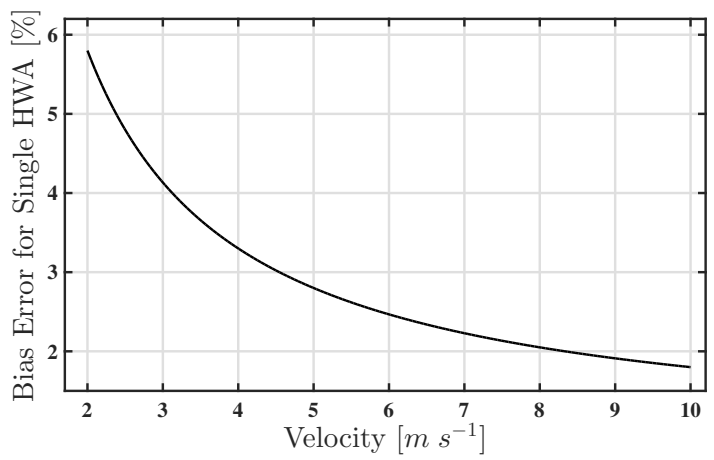

Fig. 3 Bias error calculated as function of the reference flow velocity.

\subsection{Inflow Characterization}

As aforementioned, two different boundary layers were simulated in the wind tunnel. For the smooth-wall boundary layer (see Fig. 4b) no roughness elements were added to the test section so that the free surface provides a moderate boundary-layer growth. On the other hand, for the rough-wall boundary layer the test section $(12 \mathrm{~m})$ was equipped with 95-mm high cups until $3 \mathrm{~m}$ upstream of the model after which the transition blocks are used in order to ensure flow stability (see 
a)
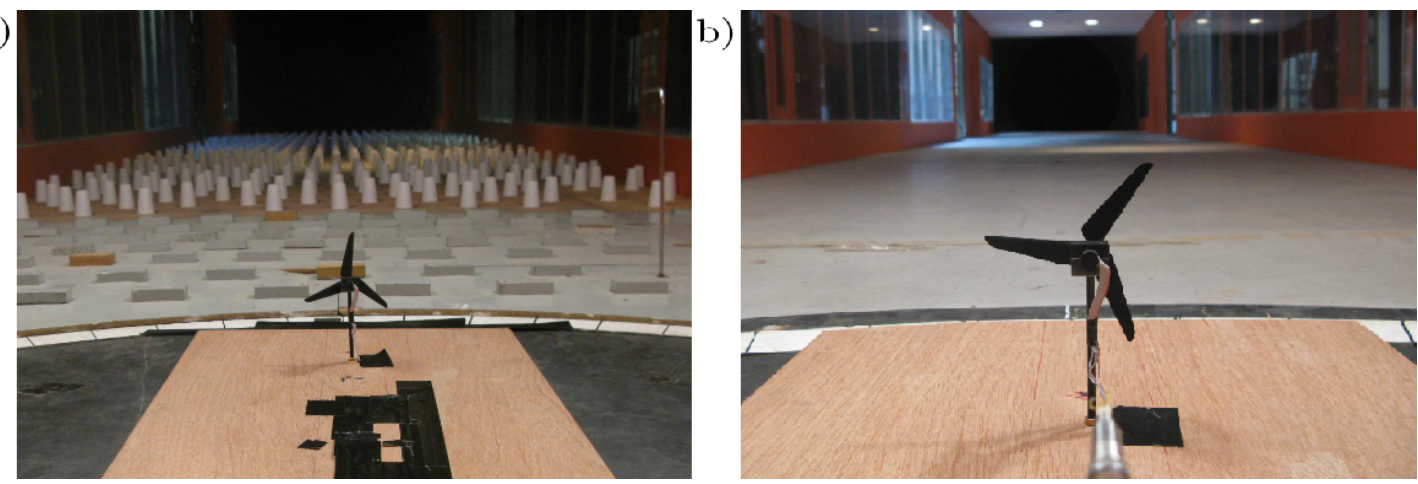

Fig. 4a). Here, a brief characterization of the two different inflow conditions is carried out, namely: the aerodynamic roughness, the turbulence intensities and the length scales are investigated.

Fig. 4 Photos from two set-ups of the wind tunnel; a) Rough-wall boundary layer, b) Smooth-wall boundary layer

The friction velocity $\left(u_{*}\right)$ was calculated via skin friction coefficient $\left(C_{f}\right)$. By plotting the mean wind speed versus height in logarithmic scale, one can use the slope of the linear curve in the logarithmic layer $(\gamma)$ in order to calculate skin friction coefficient using the expression $C_{f}=2 \cdot\left(\gamma / 2.5 \cdot U_{\infty}\right)^{2}$. The aerodynamic roughness length $\left(z_{0}\right)$ was then calculated using

$$
\frac{U_{r e f}}{u_{*}}=\frac{1}{\kappa} \cdot \ln \left(\frac{z}{z_{0}}\right)
$$

where $U_{\text {ref }}$ is the reference velocity, $u_{*}$ is the friction velocity, $\kappa$ is the von Karman constant equal to $0.41, z$ is the reference height. In addition, the power-law relationship was calculated from

$$
\frac{U}{U_{\text {ref }}}=\left(\frac{z}{z_{\text {ref }}}\right)^{\alpha}
$$

where $\alpha$ is the power-law exponent, known as the Hellmann coefficient, $z_{r e f}$ and $U_{r e f}$ are the reference height and velocity, respectively. The exponent was calculated with the values that were measured at the lowest and the highest locations where the blade tip passes. The results yield friction velocities $\left(u_{*}\right)$ of $0.56 \mathrm{~m} \mathrm{~s}^{-1}, 0.30 \mathrm{~m} \mathrm{~s}^{-1}$; values of aerodynamic roughness length $\left(z_{0}\right)$ 0.4-mm, 0.018-mm and the power-law exponents $(\alpha)$ of 0.3 and 0.16 for the two cases which are referred as the rough case and smooth case, respectively. Figures $5-7$ show the mean velocities $(\bar{U}$, $\bar{V}, \bar{W})$ and standard deviation profiles $\left(\sigma_{U}, \sigma_{V}, \sigma_{W}\right)$ normalized with the hub-height speed $\left(U_{h}\right)$ for all three velocity components; in the streamwise $(x)$, spanwise $(y)$ and vertical $(z)$ directions, respectively. In addition, the length scale distributions $\left(L_{u}, L_{v}, L_{w}\right)$ were depicted with the same coordinate system. They were calculated via autocorrelation in time and the temporal information was transformed into spatial by Taylor's hypothesis which postulates that the mean velocity is the convection velocity of the turbulence. They are non-dimensionalized with respect to the turbine diameter $(0.15 \mathrm{~m})$, since this will be relevant for the following sections. 

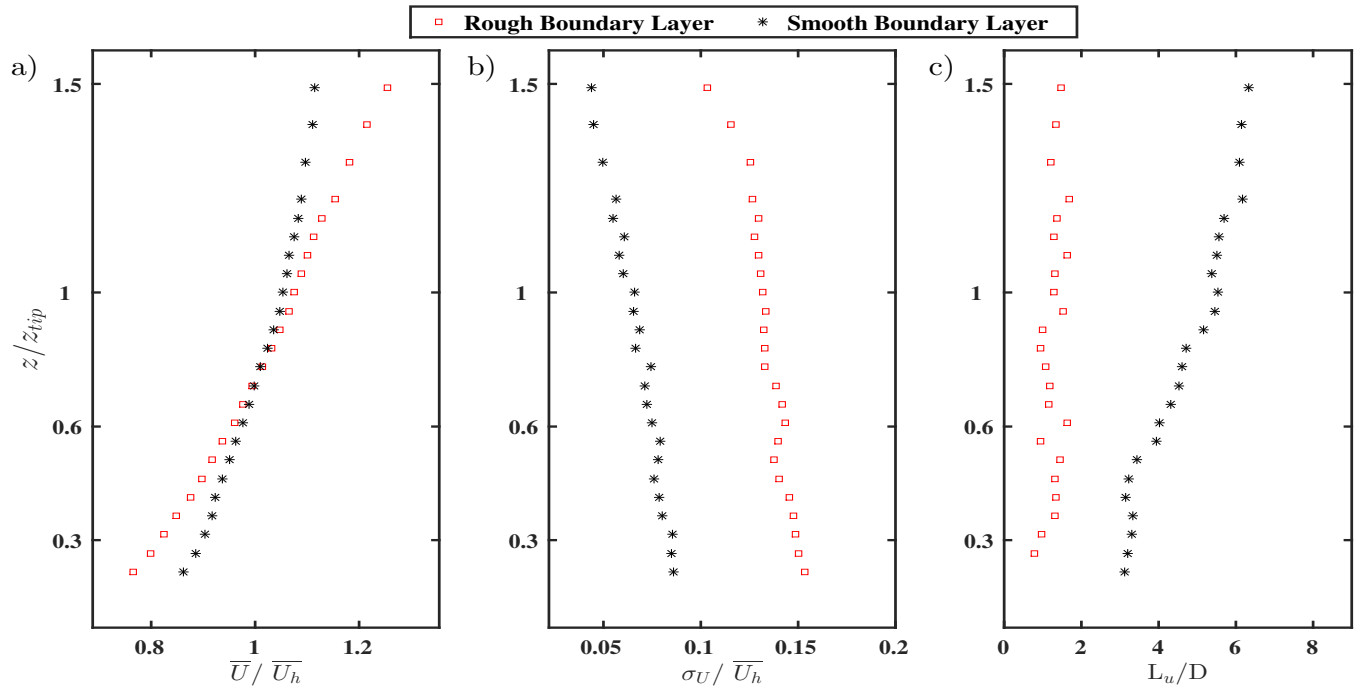

Fig. 5 a) Streamwise velocity component normalized with the hub-height wind speed. b) Streamwise turbulence intensity c) Streamwise turbulent length scales normalized with the turbine diameter. For all plots, squares represent rough case inflow conditions and stars represent smooth case inflow conditions

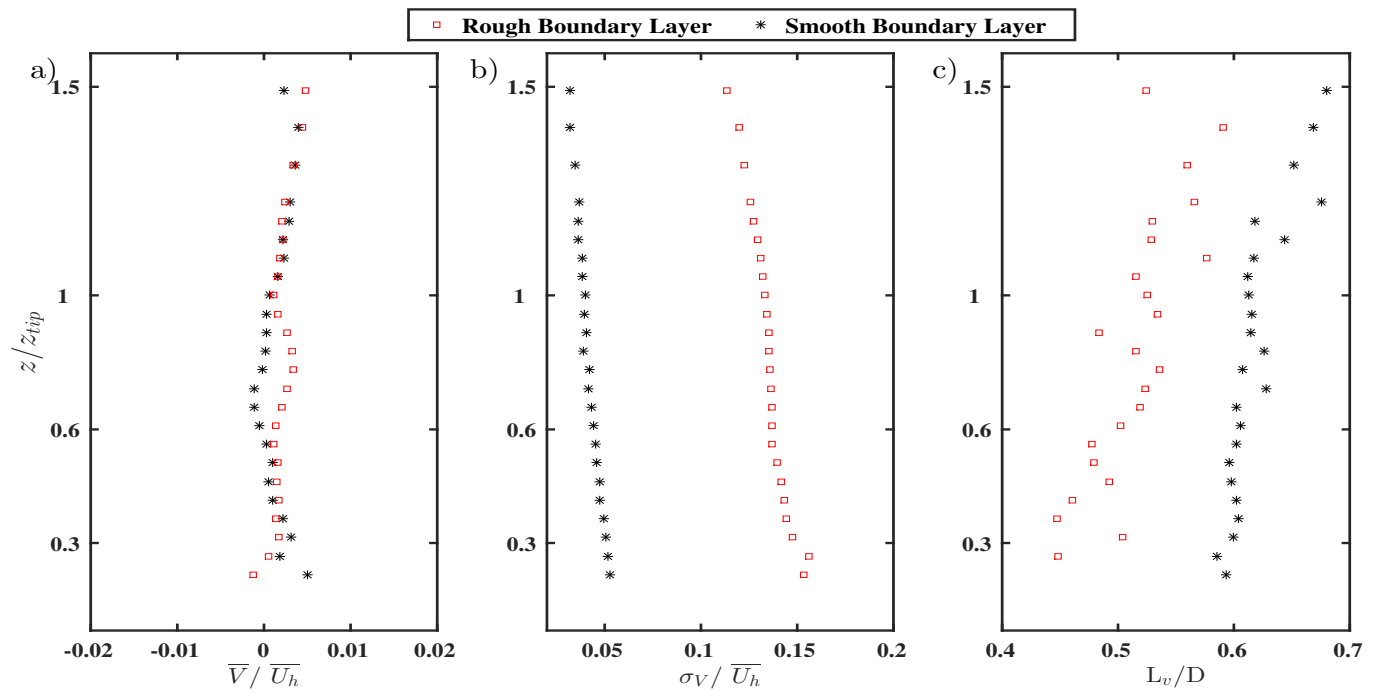

Fig. 6 a) Spanwise velocity component normalized with the hub-height wind speed. b) Spanwise turbulence intensity c) Spanwise turbulent length scales normalized with the turbine diameter. For all plots, squares represent rough case inflow conditions and stars represent smooth case inflow conditions

The streamwise velocity component for the smooth case has higher gradients but lower turbulence levels, while this is the opposite for the rough case. Additionally, it is observed that neither of the streamwise velocity component profiles reach the free stream. This ensures that the turbine models will be fully immersed in the boundary-layer flow. The earlier work on boundary-layer modelling at the L1-B wind tunnel by Conan (2012) shows that the boundary-layer depths for both cases are higher than $0.5 \mathrm{~m}$. With these two configurations the turbine model will be exposed to different shear-flow conditions. Similar comparative studies are found in the literature, in which the 

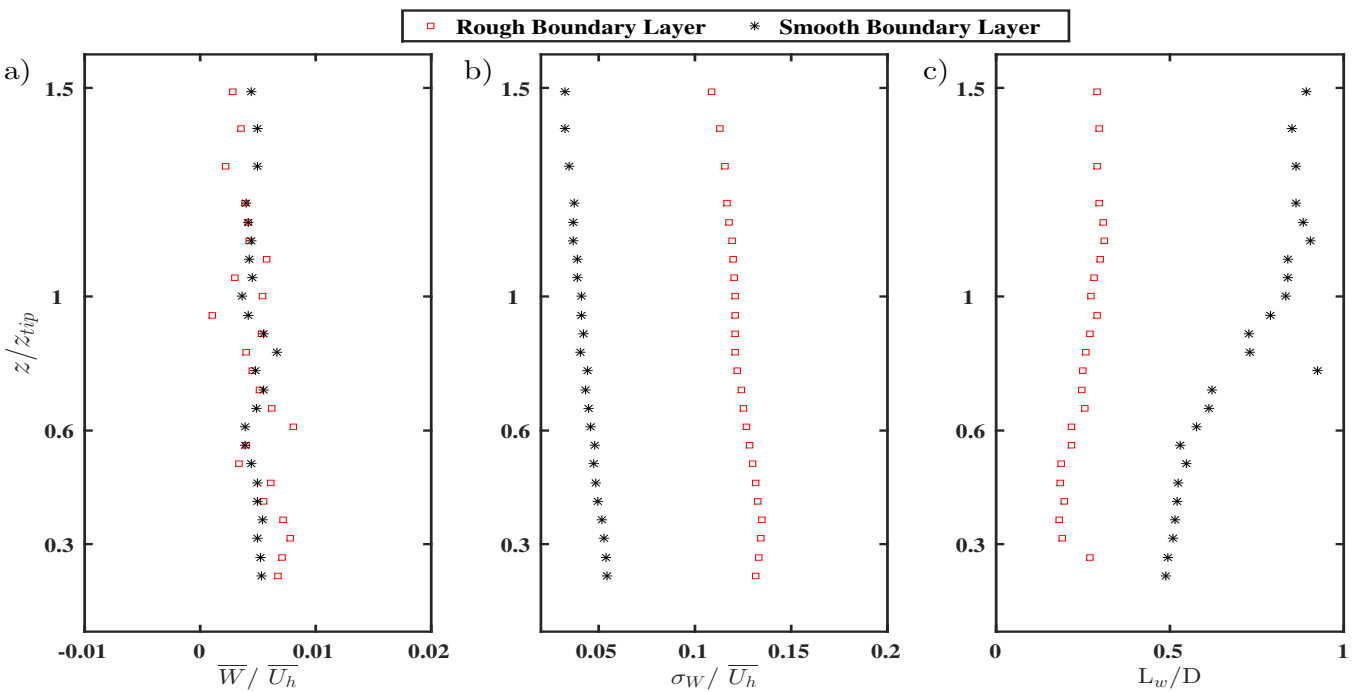

Fig. 7 a) Vertical velocity component normalized with the hub-height wind speed. b) Vertical turbulence intensity c) Vertical turbulent length scales normalized with the turbine diameter. For all plots, squares represent rough case inflow conditions and stars represent smooth case inflow conditions

experiments were conducted by keeping the hub-height speed same. This was indeed the case for this present experimental campaign. Regarding the turbulent length scales distribution, it is seen that both cases contain large scales in comparison to the turbine diameter. However, structures of the rough case are much smaller, as they are 'destroyed' by the cups located in the upstream part of the test section.

Additionally, the longitudinal length scales were compared to Counihan's empirical expression; $L_{U} x=B * z^{m}$ where $B$ and $m$ are roughness dependent parameters (Counihan, 1975). The results (see Fig. 8) show that the scales in the wind tunnel follow the correct trend for two different scaling factors.

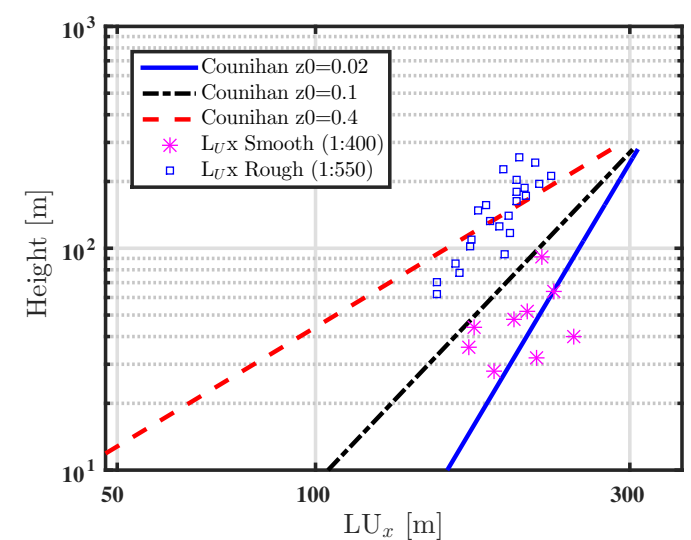

Fig. 8 Comparison of longitudinal length scales for the two different wind tunnel configurations and the corresponding empirical expression in Counihan (1975) 
Furthermore, the development of the flow within the test section was investigated. Particularly for the rough case it is important that the flow without the model does not go through a significant change along the test section. In order to illustrate this streamwise velocity component and turbulence intensity profiles at the beginning $(x=0)$ and the middle of the test section $(x=1.4 \mathrm{~m})$ is shown in Fig. 9. The measurements were carried out in earlier work (Conan, 2012) and normalization was done with the wind speed at $90-\mathrm{cm}$, represented as $U_{90}$. The results show a reasonable fit to each other. The maximum variation of turbulence intensity was calculated as $7.5 \%$ and this value was even lower for the streamwise velocity component. Considering that the furthest measurement point corresponds to $1.8 \mathrm{~m}$ far from the beginning of the test section, it was concluded that this study is a correct representation of the boundary layer effects.

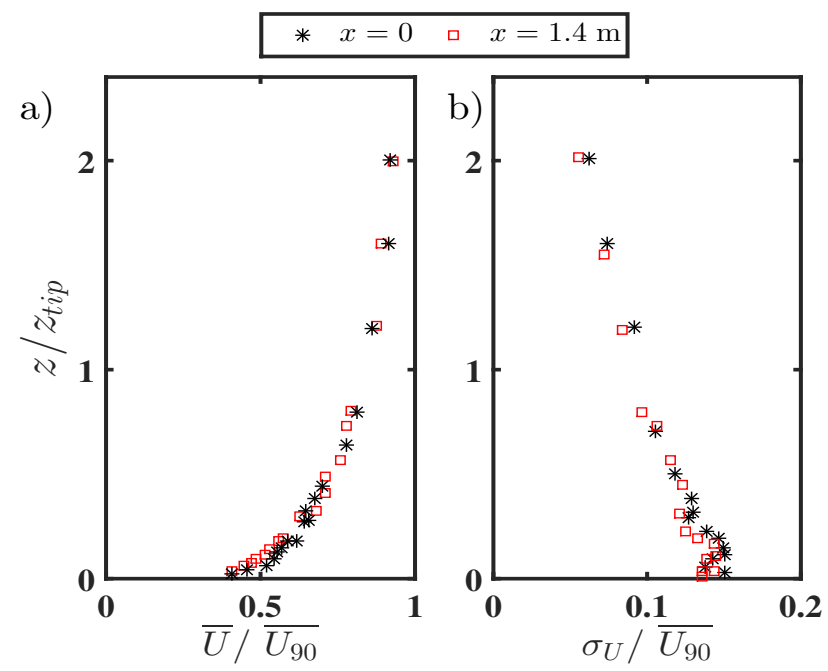

Fig. 9 a) Streamwise velocity component and b) Streamwise turbulence intensity at the beginning $(x=0)$ and the middle $(x=1.4 \mathrm{~m})$ of the centerline of the wind tunnel turntable normalized at $z=0.9 \mathrm{~m}$.

\section{Results and Discussion}

\subsection{Wake Dynamics: Velocity and Turbulence Evolution}

The spatial distribution of the streamwise velocity component at the mid-vertical plane of the wake is shown in Fig. 10. The profiles at the upstream of the turbine was obtained during the inflow characterization without the turbine. The results clearly indicate an earlier wake recovery for the rough case, where the incoming turbulence levels are higher. However, the wake deficit does not lose its effect entirely as far as $11 D$ for neither of the cases (see Fig. 11). It is clear that the streamwise velocity component distribution at the wake is not axisymmetric. This is expected as the incoming flow is not axisymmetric either. On the other hand, it is interesting to notice that for the rough case, the wake deficit at the region lower than the hub height recovers earlier in comparison to the region higher than the hub height. This is contradictory with the recently published work on analytical wake modelling with Gaussian approach by Bastankhah and Porte-Agel (2014) in which an axisymmetric wake deficit profile is assumed. Nevertheless, axisymmetry is indeed present for the smooth case. 


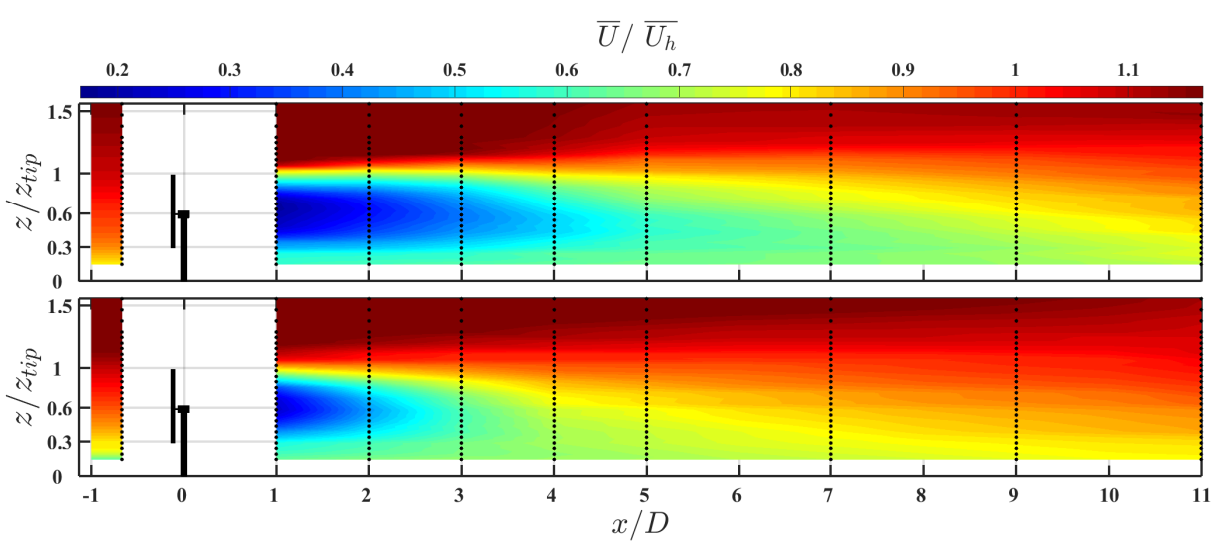

Fig. 10 Averaged streamwise velocity component at the mid-vertical plane of the wake normalized with the hub-height speed. Black dots represent the measurement points. Top: Smooth Wall Case. Bottom: Rough Wall Case.
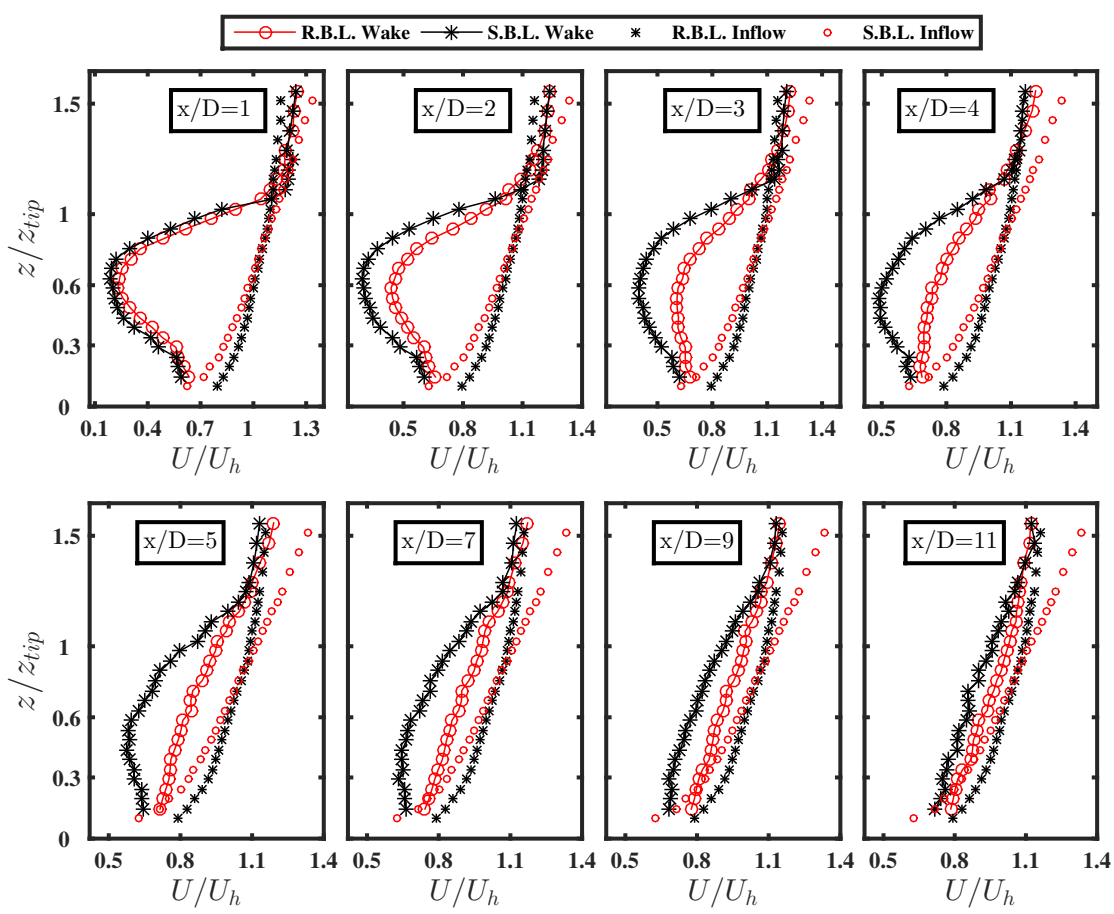

Fig. 11 Averaged streamwise velocity component profiles normalized with the hub-height speed. Continuous starred line: Smooth case wake. Stars: Smooth case inflow. Continuous circled line: Rough case wake. Circles: Rough case inflow.

As aforementioned, the mean speed is not the only parameter of interest. The increased turbulence levels may result in increased fatigue due to the loading variations along the blade span or early failure of pitch and yaw mechanisms. The turbulence intensity levels of both cases are shown 
in Fig. 12. Common point for both cases is that the highest values are reached around the tip region where the strong shear contribution to the turbulence production is significant. On the other hand, the high level of turbulence is spread to a larger region for the rough case and the peak values are reached at around 1-2D downwind distance, while this is further downstream for the smooth case $(3-4 D)$. This issue is elaborated via the shear stress distribution later on (see Fig. 13).

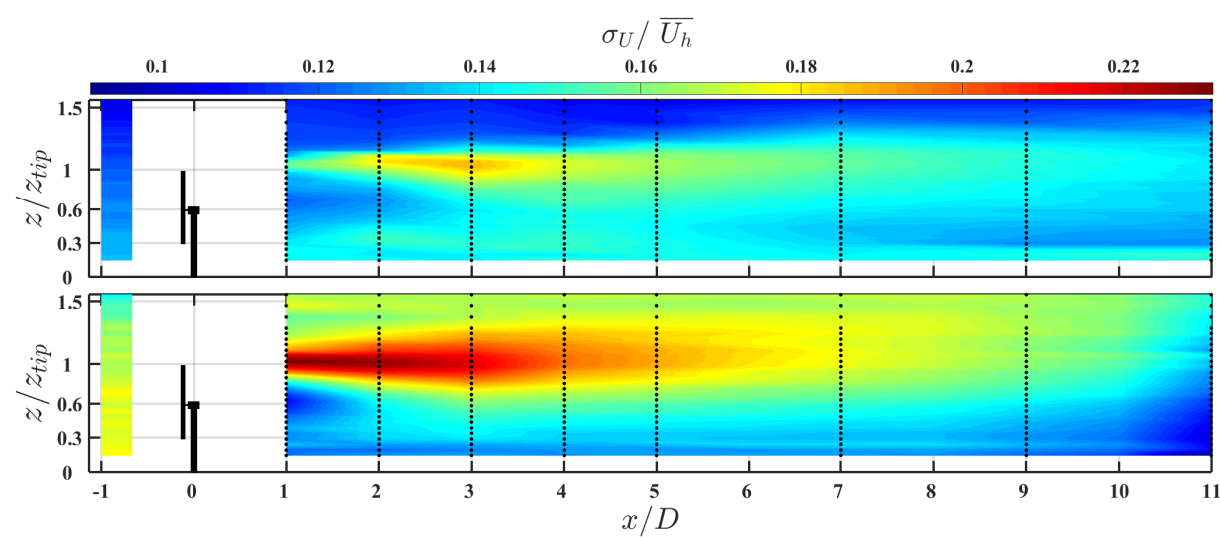

Fig. 12 Stream-wise Turbulence Intensity $\left(\sigma_{u} / \overline{U_{h}}\right)$. Top: Smooth wall case. Bottom: Rough wall case.

Additionally the evolution of the maximum added turbulence was investigated. The added turbulence is defined as $I^{+}=\sqrt{I_{\text {wake }}^{2}-I_{\text {inflow }}^{2}}$ where $I_{\text {wake }}$ and $I_{\text {inflow }}$ are the turbulence levels at the wake and at the incoming flow, respectively. It is a common procedure to seek a relationship between the decay of the maximum $I^{+}$values and the downwind distance from the turbine in meters. This study yielded with the order of $x^{-0.5}$ and $x^{-0.6}$ for the smooth case and rough case respectively. Since the added turbulence decay will be quicker with a faster wake recovery, the results are in agreement with the flow physics. Moreover, these values were in the range of the similar studies by Ainslie (1988) and Chamorro et al. (2009) in which the power coefficients were $-2 / 3$ and from -0.3 to -0.5 , respectively

Another important concept for wake dynamics is the kinematic shear stresses $\left(-\overline{u^{\prime} w^{\prime}}\right)$ that give insight to the momentum transfer in the flow. The results (see Fig. 13) show that these stresses have positive values above the tip region where the transfer takes place from the higher momentum regions of the boundary layer (above wake) towards the wake centre. Similarly, in the bottom region these stresses are negative which stands for the upwards momentum transfer from the lower region of the boundary layer again to the core of the wake. There is ongoing work to relate this energy transfer to the power produced by turbines for a fully developed wind turbine boundary layer which occurs after a sufficient number of turbines (Newman et al. 2013). Even though the trend is the same for both of the boundary-layer cases, the magnitude is higher for the rough case, which is associated with higher turbulent fluxes. Another distinguishing feature is that the highest values of the stresses are reached at $2-3 D$ for the rough case and $4-5 D$ for the smooth case. These two statements are in agreement with the wake recovery and its dynamics for various atmospheric conditions. It is observed that as the wake develops downstream, the stress levels become lower however, their effect is visible in a wider region which is also expected, considering the wake expansion and the wake deficit. 


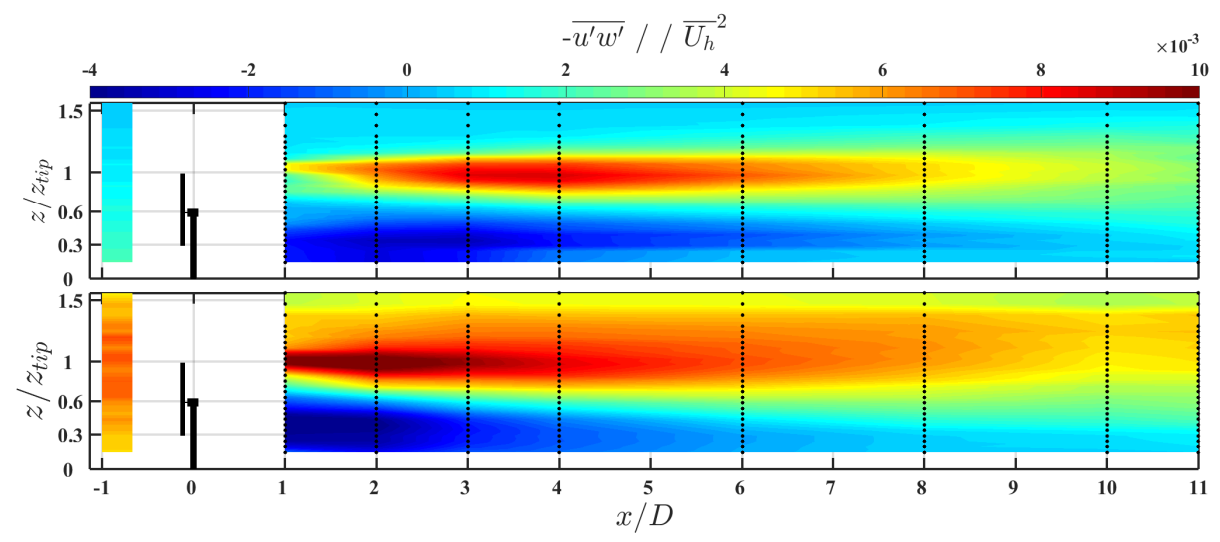

Fig. 13 Kinematic shear stresses $\left(\overline{-u^{\prime} w^{\prime}} / \mathrm{U}_{h}^{2}\right)$ for two different boundary layer configurations. Top: Smooth wall case. Bottom: Rough wall case.

\subsection{Near Wake Spectral Content}

An interesting study on the spectral analysis with a similar set-up to the present work was carried out by Chamorro et al. (2012). It was suggested to use the evolution of the turbulent length scales in order to conceptualize and model the turbine as an active filter. This means that in certain regions the turbine is able to generate or amplify some frequencies, while damping some others. The results of the present study are in line with this concept. To illustrate this, spectra at three measurement points, namely: below the hub, at the tip and above 1.5 times the tip height, are shown in Fig. 14 where the rough case and the smooth case spectra are also compared.

It is observed that for both cases, the very-large scale structures at the incoming flow that characterize the turbulent boundary layer are indeed destroyed in the area below the nacelle, with a cut-off frequency of $f / f_{t} \approx 0.09$ where $f_{t}$ is the turbine rotational frequency. Therefore, the analogy of the turbine being a high pass filter in this region can still be valid with a minor change of the cut-off frequency since this value is around 0.1 in Chamorro et al. (2012). On the other hand, not all of the large scales are fully broken down for the smooth case. The turbine distinctly excites a specific low frequency at the regions below hub and around tip. This low frequency, which is believed to be caused by the wake meandering, will be investigated in a more detailed manner in the following section. In addition to this large scale motion excited by the turbine, there is a wide range of small scales where the wake spectrum has higher energy in comparison to the incoming flow. This points out that the turbine generates turbulence mostly associated with smaller scales.

Regarding the rough case, it is observed that the added energy is much smaller than the smooth case and it is spread to all the scales. None of the distinct peaks are visible, including the turbine frequency even in the very near wake. This is due to the fact that in this wind tunnel configuration the wake behind a single turbine is extremely dissipative. The tip vortices cannot keep their integrity even up to $1 D$. Additionally, the low frequency peak is not visible either. Two possible explanations were given as to why the low frequency peaks were not visible for the rough case; either the large scales in the incoming flow (dimensions around $1.5 \mathrm{D}$ ) do not have the capability of triggering such a phenomenon, or the wake itself is so dissipative that in-fact there is ' $n o t h i n g$ to meander'. The former one was extensively studied by Devinant et al. (2011). They observed that meandering is very important when the incoming flow turbulence length scales are larger than the wake width.

Figure 14 concentrates on the three regions only in the very near wake, $(x \leq 1 D)$. Since every point measurement has one energy spectrum for each component, when all the spectra of one 

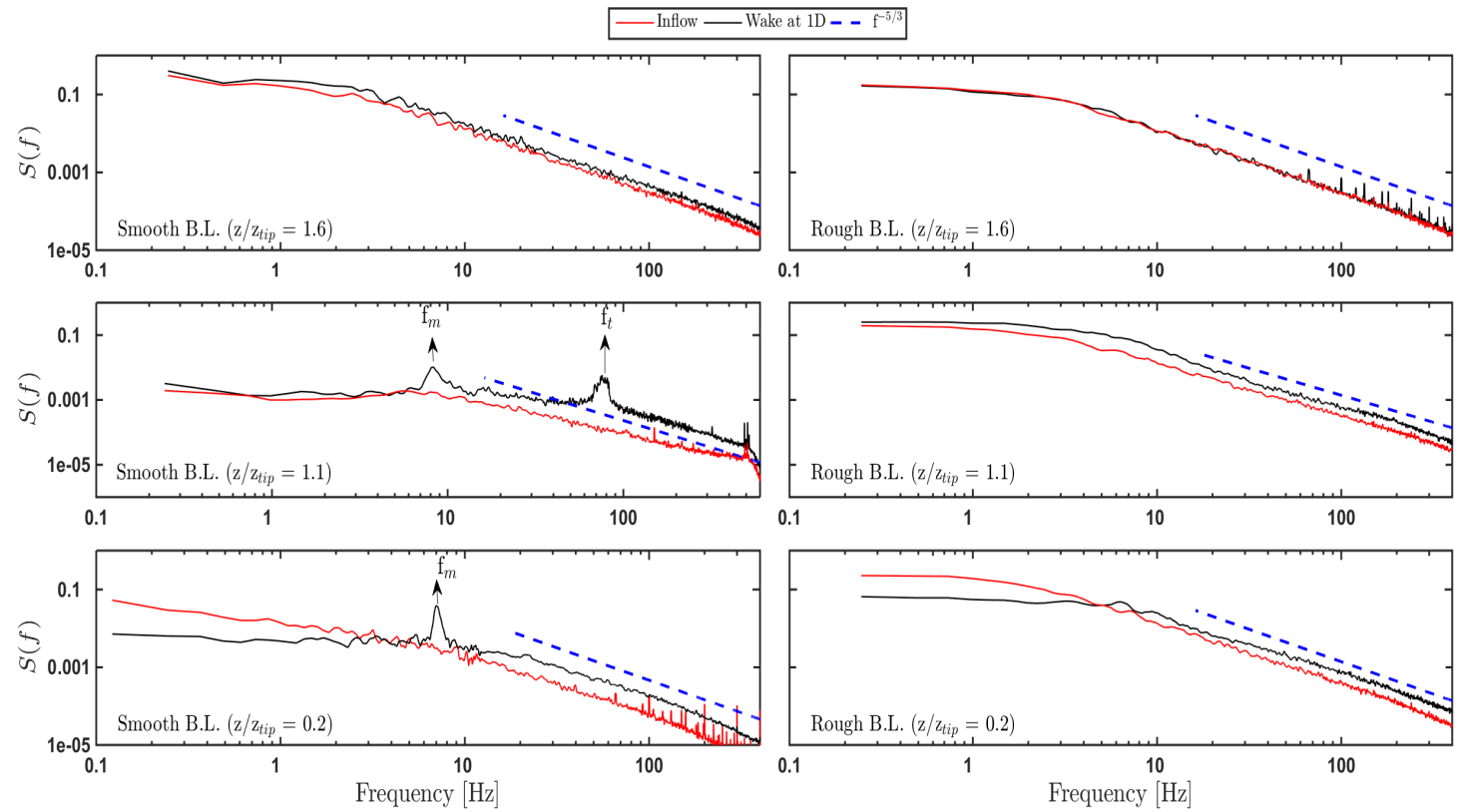

Fig. 14 Frequency spectra of the streamwise velocity component for the inflow and the wake at 1D, for two different atmospheric conditions at three specific regions. The inflow From left to right: Smooth and rough cases. From top to bottom: Measurements at above tip, tip and below hub.

component are gathered in a plot, it yields a contour. These contours for two different cases are depicted in Figs. 15 and 16.

It is observed that for the smooth case in the near wake region the turbine's signature is visible. However, it is worthwhile to mention that the blade passage frequency $\left(3 f_{T}\right)$ peak was not seen in the results. Only the turbine rotational frequency $\left(f_{T}\right)$ was detected, even though the sampling frequency was sufficient to capture both. One reason for this can be the vortex wandering. From the instantaneous particle image velocimetry (PIV) results, it was clearly seen that the vortices move up and down, therefore it might be difficult to measure each of its signals with point measurements like HWA. Another reason could be that due to the high rotational speeds, the rotor-motor mounting becomes off-centred. Hence the blades themselves are moving up and down in addition to the normal rotation. Either way, from the spectrogram this peak is only visible up to $2 D$. Afterwards most of the energy in the small scales are shifted towards the large scales. The large scales persist up to $11 D$ where the last measurement location is. Overall, the far wake is dominated by large scales and a full recovery towards the incoming flow is not visible up to the last measurement point.

For the rough case only 4 different downstream positions were considered, namely $x / D=1,4$, 7, 11 (see Fig. 15), since the other positions did not show distinctive changes. It is observed that the turbine effect is relatively less persistent. The cut-off filter characteristic is seen for the regions below hub up to $4 D$. In addition, the added energy levels are smeared across all the length scales, however, they are consistently lower in comparison to the smooth case. At the very far wake, where $x / D=11$, the turbine signature is not visible at all. 

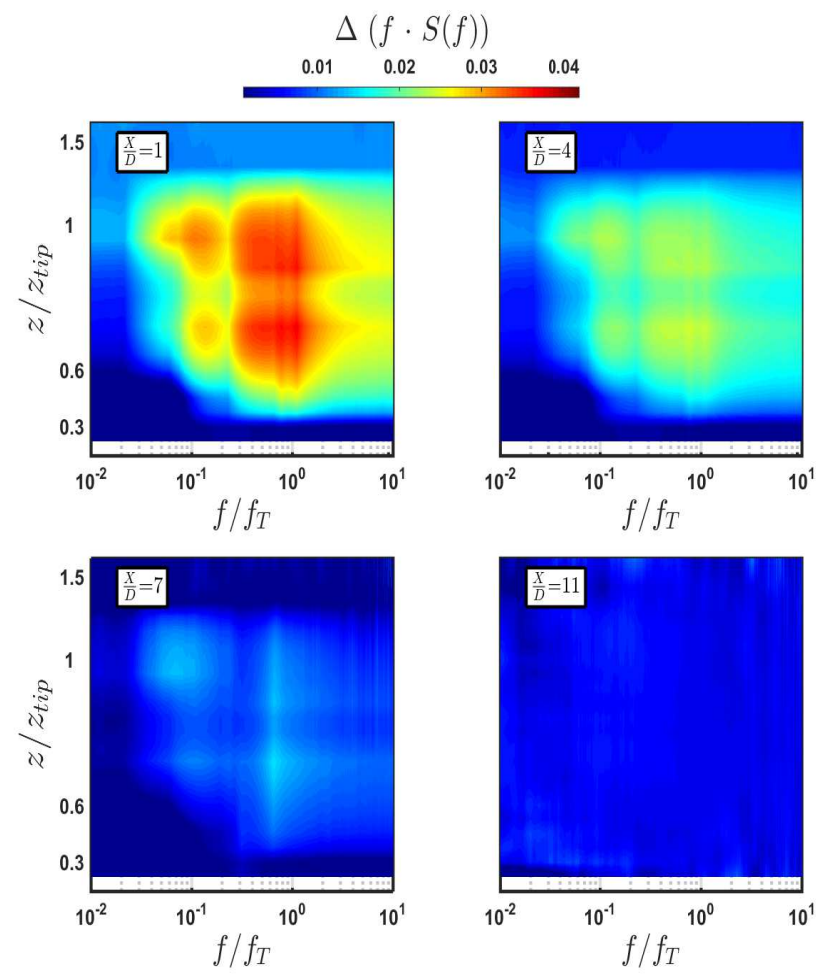

Fig. 15 Differential energy spectra obtained from each point measurement at various downstream positions for rough case. The colour scale represents the inflow subtracted wake spectrum.

\subsection{Meandering for Smooth Wall Case}

In this part of the paper, the meandering was addressed through a set of experiments only under the smooth-wall boundary-layer conditions, where a distinct low frequency peak was detected during the spectral analysis. In order to ensure that the peak's existence was persistent, the experimental campaigns were carried out at various turbine operational conditions, namely tip-speed ratios and incoming hub-height wind speeds. A number of tip-speed ratios ranging from 4.9 to 7.5 and a number of velocities ranging from $4 \mathrm{~m} \mathrm{~s}^{-1}$ to $12 \mathrm{~m} \mathrm{~s}^{-1}$ were covered. The results show that (see Fig. 17) the rotor diameter based Strouhal number when non-dimensionalized with the hub-height wind speed remained on the order of 0.25. $\left(S t=f \cdot D / U_{h}\right)$

This number is indeed in the order of bluff body vortex shedding. An experimental work on vortex shedding behind cylinders under turbulent flow was carried out by Cheung and Melbourne (1983). The data that is closest to the present study was the one with an ambient turbulence intensity of $6.8 \%$ and a Reynolds number of 80,000, which yielded a Strouhal number of 0.25 , as was found here in this work. Also another study on bluff bodies with shear flow (Maull and Young, 1973), show a similar Strouhal number when the centre-line velocity is used for the nondimensionalization of frequency. On the other hand, the article (Medici and Alfredsson, 2006) in which the meandering is linked to the bluff body vortex shedding shows a lower Strouhal number, i.e. $S t \approx 0.13$ which is in better agreement with a solid disc vortex shedding. With this output another question was raised regarding the link between the meandering phenomenon and the contribution of the wind turbine intrinsic behavior. 

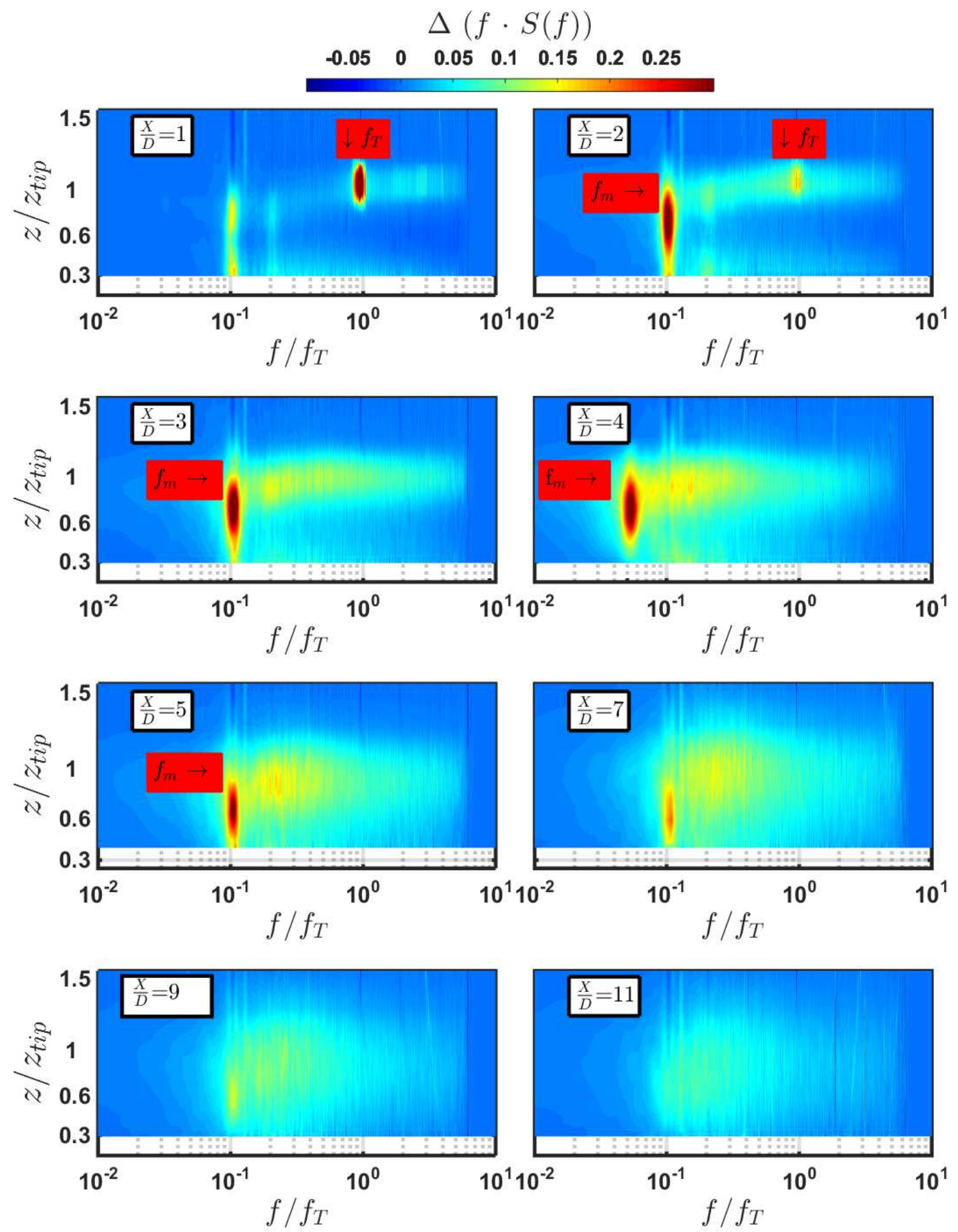

Fig. 16 Differential energy spectra obtained from each point measurement at various downstream positions for smooth-wall case. The colour scale represents the inflow subtracted wake spectrum. 


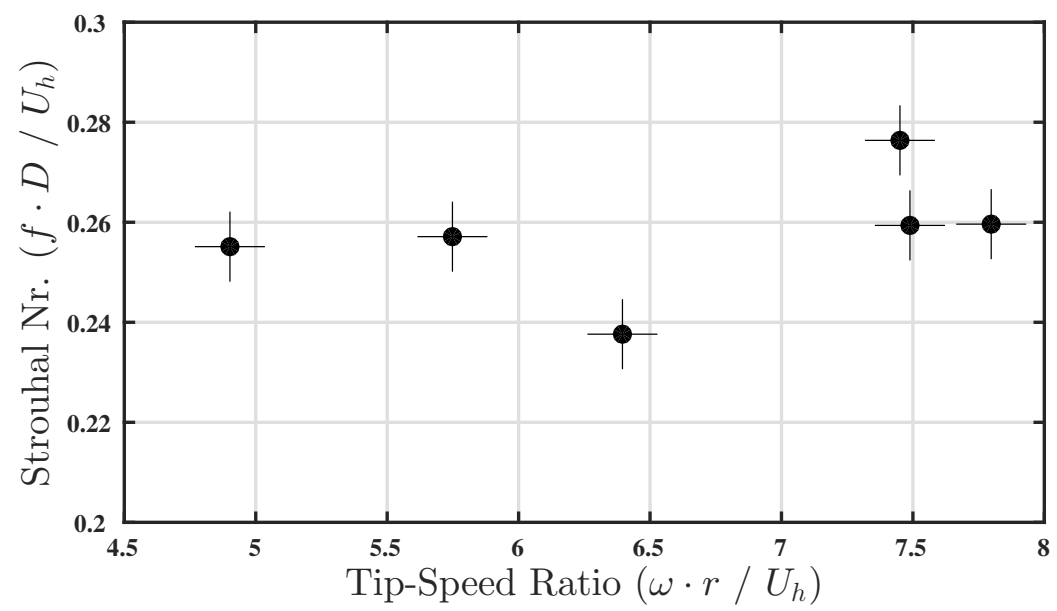

Fig. 17 Strouhal number $\left(f \cdot D / U_{h}\right)$ variation at various turbine running conditions

\section{Summary}

A wind-tunnel study was carried out with scaled three-bladed wind-turbine models under two different boundary-layer inflow conditions. Time-resolved measurements were obtained, covering $11 D$ in the streamwise and $2.5 D$ in the vertical direction. The emphasis was on the development of the streamwise velocity component and the spectral content.

The results were in agreement with the aforementioned previous works and flow physics. The higher mixing rates caused by the high incoming turbulence, accelerates the wake recovery. While this is an advantage for the downstream turbines from the wind speed point of view, the highly fluctuating wind could result in an earlier failure of the turbine components in real life situations. Therefore, the overall effect on the cost of energy can be non-beneficial. Relatively high turbulence intensity levels were observed at the top-tip region, produced by the high velocity gradients. The distinguishing feature of the turbulent shear stresses as well as the turbulence intensity spatial distribution was that the peak values were reached much nearer to the turbine for the rough case. This fits with the earlier wake recovery concept, since these are associated with the momentum transfer from the higher momentum regions towards the wake centre.

Additionally, the spectral analysis yielded three regions in the near-wake where the turbine has a different effect on the turbulent scales. As pointed out by Chamorro et al. (2012), this can be a way to parametrize the turbine from a spectral perspective, that could eventually lead to simpler wake models with higher accuracy and better physical understanding. Moreover the specific low frequency peak, which was detected around the rotor region, was attributed to the meandering phenomenon. Further investigation on this issue was carried out through a number of test campaigns, by changing the tip-speed ratio (ranging from 4.9 to 7.5 ) and the incoming velocities (ranging from $4 \mathrm{~m} \mathrm{~s}^{-1}$ to $12 \mathrm{~m} \mathrm{~s}^{-1}$ ). It was found that the rotor diameter based Strouhal number, when non-dimensionalized with the hub-height wind speed, was unconditionally on the order of 0.25 . This is an important outcome of this study. It is well known that certain contradictions exist in the literature for association of bluff body dynamics to wind turbine wake meandering. However, considering that many high-fidelity flow simulation outputs show persistent meandering deep in the wind farms, where relatively smaller scale structures are present, it is likely that the continuation of the meandering is provided via the intrinsic wind turbine behaviour. During this set of experiments this behaviour was observed for single wind turbine model wake, under controlled wind conditions. 
311

\section{References}

Ainslie J (1988) Calculating the flowfield in the wake of wind turbines. J Wind Eng Ind Aerodyn 27:213-224

Alfredsson P H, Dahlberg J D (1979) A Prelimenary Wind Tunnel Study of Windmill Wake Dispersion in Various Flow Conditions. The Aeronautical Research Institute of Sweden

Bastankhah M, Porte-Agel F (2014) A new analytical model for wind-turbine wakes. Renewable Energy 70:116-123

Bingol F, Mann J, Larsen G C (2010) Light detection and ranging measurements of wake dynamics. Part 1: one-dimensional scanning. Wind Energy 13: 5161

Bossuyt J (2013) Study of wind farm layout via wind tunnel testing. M.Sc. Thesis KU Leuven

Buckingham S (2010) Wind park siting in complex terrains assessed by wind tunnel simulations. Diploma Course Report, von Karman Institute

Chamorro L P, Porte-Agel F (2009) A Wind-Tunnel Investigation of Wind-Turbine Wakes: Boundary-Layer Turbulence Effects. Boundary-Layer Meteorol 132:129-149

Chamorro L P, Arndt R E A, Sotiropoulos F (2012) Reynolds number dependence of turbulence statistics in the wake of wind turbines. Wind Energy 15:733-742

Chamorro L P, Guala M, Arndt R E A, Sotiropoulos F (2012) On the evolution of turbulent scales in the wake of a wind turbine model. J Turbulence 13:N27

Cheung JCK, Melbourne WH (1983) Turbulence Effects on Some Aerodynamic Parameters of a Circular-Cylinder at Supercritical Reynolds-Numbers. J Wind Eng Ind Aerodyn 14(1-3):399-410

Churchfield M J, Lee S, Michalakes J, Moriarty P J (2012) A numerical study of the effects of atmospheric and wake turbulence on wind turbine dynamics. J Turbulence 13:1-32

Conan B (2012) Wind Resource Assessment in Complex Terrain by Wind Tunnel Modelling. Ph.D. thesis, VKI and Univesite d'Orleans

Counihan J (1975) Adiabatic atmospheric boundary layers: A review and analysis of data from the period 1880-1972. Atmos Environ 9:871-905

Crespo A, Hernandez J (1996) Turbulence characteristics in wind turbine wakes. J Wind Eng Ind Aerodyn 61:71-85

Crespo A, Hernandez J, Frandsen S (1999) Survey of modelling methods for wind turbine wakes and wind farms. Wind Energy 2:1-24

Devinant P, Espana G, Loyer S., Aubrun S. (2011) Spatial Study of the Wake Meandering Using Modelled Wind Turbines In a Wind Tunnel. Wind Energy 14.7:923-937

Fruytier P A (1993) Conception, experimentation, ameloration dun logiciel mde mesure anemomtrique tridimensionalles pour des sondes a trois fils chauds. M.Sc. thesis, U-VKI

Gaumond M, Rethore P-E, Bechmann A, Ott S, Larsen G C, Pena Diaz A, Hansen K S (2012) Benchmarking of Wind Turbine Wake Models in Large Offshore Wind farms. Poster at The Science of Making Torque from Wind 2012, Oldenburg, Germany.

Hancock P E, Pascheke F (2014) Wind-Tunnel Simulation of the Wake of a Large Wind Turbine in a Stable Boundary Layer: Part 2 the Wake Flow. Boundary-Layer Meteorol 151(1):23-37

Krogstad P A, Eriksen P E (2013) Blind test calculations of the performance and wake development for a model wind turbine. Renewable Energy 50:325-333

Larsen G C, Madsen H Aa, Thomsen K, Larsen T J (2008) Wake Meandering: A Pragmatic Approach. Wind Energy 11.4:377-395

Maeda T, Kamada Y, Murata J, Yonekura S, Ito T, Okawa A, Kogaki T (2011) Wind Tunnel Study on Wind and Turbulence Intensity Profiles in Wind Turbine Wake. J Therm Sci 20(2):127-132

Maull D J, Young R A (1973) Vortex shedding from bluff bodies in a shear flow. J Fluid Mech Vol 60(2): 401-409

Medici D, Alfredsson P H (2006) Measurements On a Wind Turbine Wake: 3D Effects and Bluff Body Vortex Shedding. Wind Energy 9.3:219-236 
Newman J, Lebron J, Meneveau C, Castillo L (2013) Stream-wise development of the wind turbine boundary layer over a model wind turbine array. J Phys 25:8

Odemark Y (2014) Wind-turbine wake flows - Effects of boundary layers and periodic disturbances. Technical Reports from Royal Institute of Technology, KTH Mechanics

Okulov V, Sørensen J N (2007) Stability of Helical Tip Vortices In a Rotor Far Wake. J Fluid Mech 576: $1-25$

Rados K G, Prospathopoulos J M, Stefanatos N C, Politis E S, Chaviaropoulos P K, Zervos A (2009) CFD modeling issues of wind turbine wakes under stable atmospheric conditions. Poster Session EWEC

Sanderse B, van der Pijl S P, Koren B (2011) Review of computational fluid dynamics for wind turbine wake aerodynamics, Wind Energy 147:799-819

Sarmast S, Dadfar R, Mikkelsen R, Schlatter P, Ivanell S, Sorensen J N, Henningson D S (2014) Mutual inductance instability of the tip vortices behind a wind turbine. J Fluid Mech 755

Sumer B. M., Fredsøe J (1997) Hydrodynamics around cylindrical structures, World Scientific Publishing

Troldborg N, Sørensen J N, Mikkelsen R (2007) Actuator line simulation of wake of wind turbine operating in turbulent inflow. J Phys Conf Ser 75: 012063

Trujillo J J, Kühn M (2009) Wake Meandering Modeling for Wind Turbine Loading and Performance,Offshore Wind Farms Workshop, Roskilde

Wu Y T, Porte-Agel F (2012) Atmospheric Turbulence Effects On Wind-Turbine Wakes: An LES Study. Energies 5:5340-5362

Zhang W, Markfort C D, Porte-Agel F(2013) Wind-Turbine Wakes in a Convective Boundary Layer: A Wind-Tunnel Study. Boundary Layer Meteorol 146(2):161-179 